\title{
CLASSIFICATION DE MAHLER ET DISTANCES LOCALES
}

\section{Patrice Philippon}

\begin{abstract}
We consider the classifying set, denoted $\mathbf{C} / \sim$ below, introduced by Mahler and we show that it can be endowed with non-discrete, Hausdorf topologies (even local distances) based on diophantine approximation properties of (complex) numbers. We then establish several results on the pointed topological space obtained, which could be dubbed Mahler's space (of first degree). We recover an analogue of Mahler's original classification by considering local distances to the special point (that is the class of all algebraic numbers). The main ingredients used here are diophantine properties in dimension zero over $Q$ and non-standard analysis.
\end{abstract}

\section{INTRODUCTION ET NOTATIONS}

Mahler a donné, à quarante ans d'intervalle, deux approches pour classifier les nombres (complexes) selon leurs propriétés d'approximation par les nombres algébriques, nous nous intéressons ici à la seconde, introduite dans [8]. La première, qu'on trouvera détaillée dans [10, Chapter III] ou [2, Chapter 8] (voir aussi [11, Chapter II]), s'y retrouve partiellement, elle est moins riche mais plus subtile en ce qu'elle sépare systématiquement degrés et hauteurs des nombres algébriques dans la quantification des approximations. Nous reviendrons sur cet aspect ainsi que sur les généralisations aux familles de nombres $[5,13]$ dans un travail ultérieur.

Nous considérons sur $\mathbb{C}$ la relation

$$
\alpha \sim \beta \Leftrightarrow(\alpha \text { est algébrique sur } \mathbb{Q}(\beta) \text { et } \beta \text { est algébrique sur } \mathbb{Q}(\alpha))
$$

dont on vérifie aisément que c'est une relation d'équivalence. L'ensemble quotient $\mathbb{C} / \sim$ classifie donc les nombres complexes du point de vue de leur dépendance algébrique: deux nombres d'une même classe dépendent algébriquement l'un de l'autre, les nombres algébriques forment une seule et même classe et deux nombres transcendants de classes distinctes sont algébriquement indépendants. Autrement-dit, $\mathbb{C} / \sim$ est en bijection avec l'ensemble des sous-corps algébriquement clos de degrés de transcendance $\leqslant 1$ de $\mathbb{C}$. Partant de là, on souhaiterait caractériser chaque classe de $\mathbb{C} / \sim$ en fonction des

\section{Received 5 April 1993}

Ce travail a été complété au cours d'un stage de recherche à l'Institut de Technologie de Tokyo dans le cadre d'une bourse du Centre Germano-Japonais de Berlin (JGCB).

Copyright Clearance Centre, Inc. Serial-fee code: 0004-9729/94 \$A2.00+0.00. 
propriétés d'approximation des nombres qui la composent. Mahler [8] introduit à cet effet une fonction ordre pour chaque nombre transcendant et une relation d'équivalence sur ces fonctions. Ceci conduit à une partition de $\mathbb{C} /$ en une infinité non-dénombrable de sous-ensembles (voir [1], [4, Section 3]), où l'on retrouve en particulier les familles de nombres de type de transcendance (fini) fixé. Nous nous proposons ici d'élargir substantiellement cette démarche en munissant $\mathbb{C} / \sim$ d'une topologie séparée déduite d'une distance locale non standard, notée dam (voir Théorème, Section 4 ci-dessous pour les définitions). Remarquons que nous nous restreignons, ici, aux nombres complexes, mais que notre approche est susceptible de généralisations de ce point de vue également. En particulier, les considérations du présent texte se transposent telles quelles de $\mathbb{C}$ à $\mathbb{C}_{p}$.

Pour fixer les notations nous adopterons la mesure de Mahler des polynômes à coefficients complexes. Soit $P \in \mathbb{C}\left[X_{1}, \ldots, X_{k}\right]$, la mesure de Mahler $M(P)$ est définie par $M(0)=0$ et, si $P \neq 0$,

$$
\log M(P)=\int_{0}^{1} \ldots \int_{0}^{1} \log \left|P\left(e^{2 i \pi \theta_{1}}, \ldots, e^{2 i \pi \theta_{k}}\right)\right| d \theta_{1} \ldots d \theta_{k} .
$$

Nous noterons $t(P)=e .\left((\log M(P)) /(\log 2)+d^{0} P\right)$ (où $d^{0}$ désigne le degré d'un polynôme) la taille du polynôme $P \neq 0$, on peut varier cette définition en remplaçant la mesure de Mahler par d'autres quantités équivalentes. Il est donc commode d'introduire la classe $\mathcal{T}$ des fonctions sur les polynômes (à coefficients complexes) telles que pour tout $t^{\prime} \in \mathcal{T}$ et tout $k \in \mathbb{N}^{*}$ il existe un réel $c(k) \geqslant 1$ satisfaisant

$$
c(k)^{-1} \cdot t^{\prime}(P) \leqslant t(P) \leqslant c(k) \cdot t^{\prime}(P)
$$

pour tout polynôme $P \in \mathbb{C}\left[X_{1}, \ldots, X_{k}\right] \backslash\{0\}$. On remarquera qu'avec notre taille $t$ on a $t(P Q)=t(P)+t(Q)$ pour $P, Q \in \mathbb{Z}\left[X_{1}, \ldots, X_{k}\right] \backslash\{0\}$ et $t(P) \geqslant e$ si $P \neq 0, \pm 1$.

Pour $\alpha, \beta \in \mathbb{C}$ et $P \in \mathbb{Z}[X, Y] \backslash\{0\}$ nous posons

$$
\|P(\alpha, \beta)\|=|P(\alpha, \beta)| / e \cdot M(P) \cdot(1+|\alpha|+|\beta|)^{d^{0} P},
$$

on vérifie (voir [9, Lemme 1.13] par Exemple) $\|P(\alpha, \beta)\| \leqslant 1 / e$, et aussi $\|P Q(\alpha, \beta)\|=$ $\|P(\alpha, \beta)\| \cdot\|Q(\alpha, \beta)\|$. Si $P \in \mathbb{Z}[X] \backslash\{0\}$ nous définissons pareillement $\|P(\alpha)\|$, vérifiant les mêmes propriétés.

Nous notons $\mathcal{C}$ l'ensemble des fonctions sur $\mathbb{N}$ à valeurs dans $\mathbb{R}_{+}^{*}=:\{x \in \mathbb{R} ; x>0\}$ tendant vers l'infini à l'infini. Nous posons également $\mathbb{R}_{+}=:\{x \in \mathbb{R} ; x \geqslant 0\}=\mathbb{R}_{+}^{*} \cup\{0\}$ et $\overline{\mathbb{R}}_{+}=: \mathbb{R}_{+} \cup\{+\infty\}$.

Définitions: Soient $\alpha, \beta \in \mathbb{C}$ et $\varphi \in \mathcal{C}$. 
Nous appelons fonction ondre d'approximation mutuelle (logarithmique) relative i $\varphi$ de $(\alpha, \beta)$, et nous notons $O_{\varphi}(\cdot \mid \alpha, \beta): \mathbb{N} \rightarrow \overline{\mathbb{R}}_{+}$, la fonction croissante définie par

$$
O_{\varphi}(j \mid \alpha, \beta)=\max \left\{\log \log \left(\|P(\alpha, \beta)\|^{-1}\right)-\log t(P)\right\}
$$

où le maximum est pris sur tous les polynômes $P \in \mathbb{Z}[X, Y] \backslash\{0\}$ sans facteur dans $\mathbb{Z}[X]$ et $\mathbb{Z}[Y]$, satisfaisant $\log t(P) \leqslant \varphi(j)$.

De même nous appelons fonction ordre d'approximation algébrique (logarithmique) relative à $\varphi$ de $\alpha$, et nous notons $O_{\varphi}(\cdot \mid \alpha): \mathbb{N} \rightarrow \overline{\mathbb{R}}_{+}$, la fonction croissante définie par

$$
O_{\varphi}(j \mid \alpha)=\max \left\{\log \log \left(\|P(\alpha)\|^{-1}\right)-\log t(P)\right\}
$$

où le maximum est pris sur tous les polynômes $P \in \mathbb{Z}[X]$ non constants, satisfaisant $\log t(P) \leqslant \varphi(j)$.

REMARQUE 1 . On vérifie, grâce aux propriétés de multiplicativité de $\|P(\alpha, \beta)\|$ (respectivement $\|P(\alpha)\|)$ et d'additivité de $t(P)$, que les maximums dans les définitions ci-dessus sont atteints pour des polynômes irréductibles. Et en prenant une puissance convenable d'un de ces polynômes irréductibles, on vérifie tout aussi bien que ces maximums sont atteints par des polynômes satisfaisants $\varphi(j)-\log 2 \leqslant \log t(P) \leqslant \varphi(j)$.

Remarque 2. Dans la notation $O_{\varphi}$, on pourra omettre l'indice $\varphi$ lorsqu'il n'y aura pas d'ambiguité sur le choix de cette fonction.

En guise de lien avec [8] et [4] rappelons que la fonction ordre d'un nombre transcendant $\alpha$ introduite par Mahler [8] peut s'écrire

$$
O_{\text {Mahler }}(u \mid \alpha)=\max \left\{\log \|P(\alpha)\|^{-1}\right\}
$$

où le maximum est pris sur $P \in \mathbb{Z}[X] \backslash\{0\}$ de taille $\leqslant \log u\left(u \in \mathbb{N}^{*}\right)$.

Soit $\varphi \in \mathcal{C}$, la limite supérieure dans $\overline{\mathbb{R}}_{+}$de la suite $\left(1+\left(O_{\varphi}(j \mid \alpha, \beta) / \varphi(j)\right)\right)_{j \in \mathbb{N}}$ ne dépend pas du choix de $\alpha$ et $\beta$ dans leurs classes respectives $\tilde{\alpha}$ et $\tilde{\beta}$ dans $\mathbb{C} / \sim$, notons la $\bar{\rho}(\tilde{\alpha}, \widetilde{\beta})$. L'ordre de transcendance de $\alpha$ n'est autre que la limite supérieure de la suite $\left(1+\left(O_{\varphi}(j \mid \alpha) / \varphi(j)\right)\right)_{j \in \mathbb{N}}$.

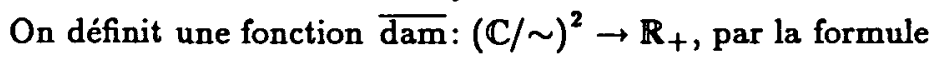

$$
\overline{\operatorname{dam}}(\tilde{\alpha}, \tilde{\beta})=2 / \tilde{\rho}(\tilde{\alpha}, \tilde{\beta}) .
$$

Cette fonction est symétrique et satisfait $\overline{\operatorname{dam}}(\tilde{\alpha}, \tilde{\alpha})=0$, mais il existe des classes $\tilde{\alpha}, \tilde{\beta}$ distinctes de $\mathbb{C} / \sim$ telles que $\overline{\operatorname{dam}}(\tilde{\alpha}, \tilde{\beta})=0$ (par exemple la classe des nombres algébriques et la classe d'un nombre de Liouville). 
Nous n'étudierons pas dans ce texte la fonction $\overline{\mathrm{dam}}$ introduite dans l'alinéa précédent. Notre propos est plutôt de la modifier de façon à obtenir une distance locale sur $\mathbb{C} / \sim$ (voir Section 4, Théorème). Pour ce faire nous devrons entre autre étoffer l'ensemble des valeurs prises autour de 0 , et nous utiliserons les hyperréels infiniment voisins de 0 . La notation "dam" rappelle qu'il s'agit d'une distance d'approximation mutuelle (locale, non standard).

Indiquons pour conclure cette introduction que la construction des hyperréels nécessite l'axiome du choix et que leur unicité (à isomorphisme près) dépend de l'hypothèse du continu, dans la suite de ce texte nous admettons l'axiome du choix.

NOTA BENE: Pour éviter l'inflation des indices nous renuméroterons à partir de 1 dans chaque démonstration les réels $c_{1}, c_{2}, \ldots$

Je remercie les arbitres pour leurs lectures minutieuses de ce texte.

\section{HYPER- ET DIHYPERRÉELS}

Décrivons brièvement pour $\mathbb{R}$ la notion non-standard de corps d'hypernombres (voir [7] ou [6]).

On se fixe une fois pour toutes un ultrafiltre $U$ sur $\mathbb{N}$ plus fin que le filtre de Fréchet des complémentaires des parties finies (voir [3, Sections 2.1-2.3]), l'existence d'un tel ultrafiltre résulte du "théorème" de Zorn (voir [3, Section 2.7]). On montre facilement que si $\mathcal{U}$ est un filtre alors $\mathcal{U}$ est un ultrafiltre si et seulement si pour toute partie $E$ de $\mathbb{N}$ on a ou bien $E \in \mathcal{U}$, ou bien $\mathbb{N} \backslash E \in \mathcal{U}$ (cette propriété caractéristique est prise comme définition des ultrafiltres dans [7, Appendice]). En particulier, l'ultrafiltre $U$ que nous nous sommes fixé étant plus fin que le filtre de Fréchet ne contient aucune partie finie, il n'est pas trivial.

On définit sur $\mathbb{R}^{\mathbb{N}}$ la relation de $\mathcal{U}$-équivalence suivante:

$$
\mathfrak{a} \sim u \mathfrak{b} \Leftrightarrow\{j \in \mathbb{N} ; \mathfrak{a}(j)=\mathfrak{b}(j)\} \in \mathcal{U} .
$$

C'est bien une relation d'équivalence et l'ensemble quotient ${ }^{*} \mathbb{R}=: \mathbb{R}^{\mathbb{N}} / \sim u$ est appelé ensemble des hyperréels. On vérifie facilement que les opérations et relations naturelles sur $\mathbb{R}^{\mathbb{N}}$ induisent une structure de corps commutatif totalement ordonné sur ${ }^{*} \mathbb{R}$ dans lequel on injecte canoniquement $\mathbb{R}$ en envoyant un réel $x$ sur la classe de l'application constante $\mathfrak{x}(j)=x(j \in \mathbb{N})$. On étend la valeur absolue de $\mathbb{R} \dot{a} * \mathbb{R}$ en notant $|a|$ l'hyperréel $\geqslant 0$ représenté par la suite $|\mathfrak{a}(j)|(j \in \mathbb{N})$ si a est représenté par $\mathfrak{a} \in \mathbb{R}^{\mathbb{N}}$. En toute rigueur il faut noter que ${ }^{*} \mathbb{R}\left(=\mathbb{R}^{\mathbb{N}} / \sim u\right)$ dépend à priori de l'ultrafiltre $U$ choisi. On peut montrer, à l'aide de l'hypothèse du continu, que * $\mathbb{R}$ ne dépend en fait pas, à isomophisme près, de ce choix (mais ce résultat n'est pas indispensable à notre exposition). En revanche, l'application $\mathbb{R}^{\mathbf{N}} \rightarrow{ }^{\mathbb{R}}$ qui, à une application associe sa classe dépend essentiellement de $\mathcal{U}$. 
On peut généraliser la construction des hyperréels (voir [7]). Plus précisément, la relation d'équivalence $\sim u$ s'étend à tout ensemble de la forme $S^{N}$ (où $S$ désigne un ensemble non vide), et on note ${ }^{*} S$ l'ensemble quotient $S^{\mathbb{N}} / \sim u$. En particulier, en prenant $S=\mathbb{R}$ on obtient un ensemble ${ }^{* *} \mathbb{R}$ que nous appelons ensemble des dihyperréels. Comme pour $\mathbb{R}$ on vérifie que ${ }^{* *} \mathbb{R}$ est un corps commutatif totalement ordonné dans lequel $\mathbb{R}$ s'injecte canoniquement. Si $a \in \mathbb{R}^{\mathbf{N}}$ notons a $\in \mathbb{R}$ l'hyperréel représenté par $a$, puisque $\mathbb{R} \subset \mathbb{R}$ on peut aussi considérer $a$ comme un élément de $\left({ }^{*} \mathbb{R}\right)^{N}$ et noter $a^{\prime} \in{ }^{* *} \mathbb{R}$ le dihyperréel défini par a. Il convient de remarquer qu'en général l'image de $a$ dans ${ }^{* *} \mathbb{R}$ n'est pas $a^{\prime}$. Par contre il n'y a pas d'ambiguité à noter d'une même lettre un réel et ses images dans ${ }^{*} \mathbb{R}$ et ${ }^{* *} \mathbb{R}$ (respectivement un hyperréel et son image dans $* * \mathbb{R}$ ) pour les injections canoniques.

On dit qu'un dihyperréel $a$ est infiniment petit (respectivement infiniment grand) si et seulement si $|a|$ est plus petit (respectivement grand) que tout réel, on note $\mathcal{M}$ l'ensemble des dihyperréels infiniment petits et $\mathcal{M}_{+}^{*}$ l'ensemble des infiniments petits $>0$. Deux dihyperréels sont dits infiniment voisins si leur différence est infiniment petite. Toute fonction $\varphi \in \mathcal{C}$ détermine un hyperréel, que nous notons aussi $\varphi$, et un dihyperréel $\varphi^{\prime}$ tels que $\varphi, \varphi^{\prime}$ et $\varphi / \varphi^{\prime}$ sont infiniment grands.

On dit qu'un dihyperréel est limité (par un réel $x$ ) s'il est borné en valeur absolue par $x$. L'ensemble $\mathcal{L}$ des dihyperréels limités est un anneau dont $\mathcal{M}$ est un idéal maximal. En fait, tout dihyperréels limité est infiniment voisin d'un réel, ce qui se traduit par l'isomorphisme $\mathcal{L} / \mathcal{M} \simeq \mathbb{R}$, on a également $\mathcal{L} \cap \mathbb{R} / \mathcal{M} \cap \mathbb{R} \simeq \mathbb{R}$. Si $a \in \mathcal{L}$ le réel dont il est infiniment voisin est appelé la partie standard de $a$, notée ${ }^{\circ} a$. Si le dihyperréel $a$ est infiniment grand positif on posera ${ }^{\circ} a=+\infty$.

D'un autre côté $1+\mathcal{M}$ est un sous-groupe du groupe multiplicatif $\left({ }^{* *} \mathbb{R}\right)_{+}^{*}$ des dihyperréels $>0$, et nous notons $\Gamma^{*}$ le groupe quotient $\left({ }^{* *} \mathbb{R}\right)_{+}^{*} /(1+\mathcal{M})$. On remarque que $\Gamma_{1}^{*}=:\left({ }^{*} \mathbb{R}\right)_{+}^{*} /(1+\mathcal{M} \cap \mathbb{R})$ s'identifie à un sous-groupe de $\Gamma^{*}$. On adjoint à $\Gamma^{*}$ les symboles 0 et $+\infty$, l'ensemble $\Gamma=\Gamma^{*} \cup\{0\}$ (respectivement $\bar{\Gamma}=\Gamma^{*} \cup\{0,+\infty\}$ ) s'écrit alors comme la réunion disjointe de $\mathbb{R}_{+}$(respectivement $\left.\overline{\mathbb{R}}_{+}\right), \mathcal{M}_{+}^{*} /(1+\mathcal{M}$ ) et $\left(\mathcal{M}_{+}^{*}\right)^{-1} /(1+\mathcal{M})$, et se trouve naturellement muni d'une relation d'ordre total. Notons $s:\left({ }^{* *} \mathbb{R}\right)_{+}^{*} \rightarrow \Gamma^{*}$ la surjection canonique, nous étendons $s$ à $* * \overline{\mathbb{R}}_{+}=:\{a \in$ $* * \mathbb{R} ; a \geqslant 0\} \cup\{+\infty\}$ en posant $s(0)=0$ et $s(+\infty)=+\infty$. Cette application $s$ peutêtre identifiée à l'application standard pour les dihyperréels limités d'inverses également limités, mais elle en différe précisément sur les infiniment petits et les infiniment grands.

Soient $\gamma, \gamma^{\prime} \in \Gamma^{*}$, par définition $\Gamma^{*}$ est un groupe commutatif dont on notera $\gamma \cdot \gamma^{\prime}$ l'opération. Remarquons que si $a, a^{\prime} \in\left({ }^{* *} \mathbb{R}\right)_{+}^{*}$ satisfont $s(a)=\gamma, s\left(a^{\prime}\right)=\gamma^{\prime}$ alors $s\left(a+a^{\prime}\right)$ ne dépend pas du choix de $a$ et $a^{\prime}$, mais seulement de $\gamma$ et $\gamma^{\prime}$, on posera $\gamma+\gamma^{\prime}=s\left(a+a^{\prime}\right) \in \Gamma^{*}$, on a $\gamma+\gamma^{\prime}=\gamma^{\prime}+\gamma$. Si $\gamma^{\prime} / \gamma \in \mathcal{M}_{+}^{*} /(1+\mathcal{M})$ on a $\gamma+\gamma^{\prime}=\gamma$, et si $\gamma_{1}=\gamma+\gamma^{\prime}$ et $\gamma^{\prime} / \gamma \notin \mathcal{M}_{+}^{*} /(1+\mathcal{M})$ on notera aussi $\gamma_{1}-\gamma=\gamma^{\prime}$. Nous ferons 
encore les conventions naturelles suivantes $\left(\gamma, \gamma_{0}, \gamma_{1} \in \bar{\Gamma}, \gamma_{0} \neq 0, \gamma_{1} \neq+\infty\right)$ :

$$
\begin{array}{cccc}
\gamma_{1} \cdot 0 & =0 . \gamma_{1}=0 & (+\infty) \cdot \gamma_{0}=\gamma_{0} \cdot(+\infty)=+\infty & \gamma_{0}-0=\gamma_{0} \\
\gamma+0=0+\gamma=\gamma & (+\infty)+\gamma=\gamma+(+\infty)=+\infty & (+\infty)-\gamma_{1}=+\infty
\end{array}
$$

Ainsi, lorsque $\gamma, \gamma^{\prime}, \gamma^{\prime \prime} \in \bar{\Gamma}$ et $\gamma \leqslant \gamma^{\prime}$ on a $\gamma+\gamma^{\prime \prime} \leqslant \gamma^{\prime}+\gamma^{\prime \prime}$.

On vérifie aisément que si $a, \mathfrak{a}^{\prime} \in\left(\left({ }^{*} \mathbb{R}\right)_{+}^{*}\right)^{N}$ satisfont $s(\mathfrak{a}(j))=s\left(\mathfrak{a}^{\prime}(j)\right)(j \in \mathbb{N})$ alors les dihyperréels définis par $a$ et $a^{\prime}$ ont même image dans $\Gamma$, autrement-dit ${ }^{*} \Gamma_{1} \subset \Gamma$ où $\Gamma_{1}=: \Gamma_{1}^{*} \cup\{0\}$.

Nous allons tirer parti de la présence d'infiniment petits dans $\Gamma$ pour modifier notre fonction $\overline{\mathrm{dam}}$ en une distance (locale) à valeurs dans $\Gamma$. Terminons ce paragraphe par un lemme technique qui nous sera bien utile au paragraphe suivant. Soit $f$ une fonction croissante de $\left({ }^{*} \mathbb{R}\right)_{+}^{*}$ à valeurs dans $\Gamma_{1}$, pour $j \in \mathbb{N}$ nous noterons $E_{j}(f)=:\{f(a) ; a \in$ $\left.* \mathbb{R}, s(a)=2^{j}\right\}$ et, si ces ensembles admettent des bornes supérieures, pour $n \in \mathbb{N}$ nous poserons $a_{n}(f)$ la suite $\left(\sup E_{j+n}(f) / 2^{j}\right)_{j \in N}$ d'éléments de $\Gamma_{1}$ et $a_{n}(f)$ l'élément de $\Gamma$ associé. Comme $f$ est croissante on a clairement $a(f)=: a_{0}(f) \leqslant a_{n}(f)$, plus généralement si $f \leqslant f^{\prime}$ on a $a(f) \leqslant a\left(f^{\prime}\right)$.

LEMME 1. Soit $f$ une fonction croissante de $\left({ }^{*} \mathbb{R}\right)_{+}^{*}$ dans $\Gamma_{1}$. Supposons d'une part $f(a) \geqslant s(a)$, et d'autre part qu'il existe $\ell \in \mathbb{N}, c \in \mathbb{R}_{+}^{*}, c \geqslant 1$ tels que, si $a_{0} \leqslant a_{1} \leqslant \ldots \leqslant a_{\ell}$ alors:

$$
\left(f\left(a_{0}\right) \leqslant c . s\left(a_{\ell}\right)\right) \quad \text { ou } \quad\left(\exists h \in \mathbb{N} ; 1 \leqslant h \leqslant \ell, f\left(a_{h}\right) \leqslant f\left(a_{h-1}\right)+c . s\left(a_{\ell}\right)\right) .
$$

Sous ces hypothèses, les ensembles $E_{j}(f)$ admettent des bornes supérieures dans $\Gamma_{1}$ et, s'il existe $y \in \mathbb{R}, y \geqslant 1$ tel que $f(y) \notin s(\mathcal{L})$, on a $a_{n}(f)=a(f)$ pour tout $n \in \mathbb{N}$.

DÉmonstration: D'après l'hypothèse il existe au plus $\ell^{\prime}\left(0 \leqslant \ell^{\prime} \leqslant \ell\right)$ éléments $a_{h} \in 2^{j} .(1+\mathcal{M})\left(h=1, \ldots, \ell^{\prime}\right)$ tels que

$$
f\left(a_{1}\right)>c . s\left(a_{1}\right) \text { et } f\left(a_{h}\right)>(c+1) \cdot f\left(a_{h-1}\right)(h \geqslant 2) .
$$

Si $\ell^{\prime}=0$ alors $s(a) \leqslant f(a) \leqslant c . s(a)$ pour $a \in\left({ }^{*} \mathbb{R}\right)_{+}^{*}$, dans ce cas on pose $A=2^{j}$ et sinon $A=f\left(a_{\ell^{\prime}}\right)$. Ainsi, pour $a \in 2^{j} \cdot(1+\mathcal{M})$ on a $f(a) / A \leqslant c+1$ et l'ensemble

$$
E_{j}^{\prime}=\left\{f(a) / A \geqslant 1 ; a \in 2^{j} \cdot(1+\mathcal{M})\right\}
$$

s'identifie à un sous ensemble non vide, borné $($ par $c+1)$ de $\mathbb{R}_{+}^{*}$. Il admet donc une borne supérieure et $A \cdot \sup E_{j}^{\prime}$ est une borne supérieure de $E_{j}(f)$.

S'il existe $y \in \mathbb{R}, y \geqslant 1$ tel que $f(y) \notin s(\mathcal{L})$ alors pour tout $b \in \mathbb{R}, b \geqslant y$ on a $f(b) \notin s(\mathcal{L})$ et l'hypothèse sur $f$ entraîne l'existence d'au plus $\ell^{\prime}+1\left(0 \leqslant \ell^{\prime}<\ell\right)$ 
éléments $a_{h}$ de ${ }^{*} \mathbb{R} \cap \mathcal{L}$ tels que $a_{\ell^{\prime}}>\ldots>a_{0}>y$ et $f\left(a_{h}\right)>f\left(a_{h-1}\right)$. On a donc $f(b)=f\left(a_{\ell^{\prime}}\right)$ pour tout $b \in{ }^{*} \mathbb{R} \cap \mathcal{L}, b \geqslant a_{\ell^{\prime}}$, et donc $a_{n}(f)=f\left(a_{\ell^{\prime}}\right) / s\left(2^{\omega}\right)$ pour tout $n \in \mathbb{N}$ et où $\omega$ désigne le dihyperréel déterminé par l'application identique de $\mathbb{N}$.

Rappelons que nous utiliserons les infiniments grands (respectivement petits) de $\Gamma$ pour affiner les notions d'ordres de transcendance et d'approximation (respectivement la fonction $\overline{\mathrm{dam}}$ ) pour les nombres tels que $\bar{\rho}(\tilde{\alpha}, \tilde{\beta})=+\infty$. Nous utiliserons le Lemme 1 et les dihyperréels pour surmonter une difficulté technique (voir Remarque 3 ci-après), à savoir qu'un sous-ensemble borné de ' $\mathbb{R}$ n'a pas nécessairement de borne supérieure dans $* \overline{\mathbb{R}}$. Grosso modo, avec les notations du Lemme 1 , les dihyperréels $a_{n}(f)$ jouent le rôle de borne supérieure des hyperréels $f(a) / s(a)$.

\section{ORDRES D'APPROXIMATION}

(A) APPROXIMATION ALGÉBRIQUE. On peut maintenant associer à chaque application $O_{\varphi}(. \mid \alpha)$ l'hyperréel qu'elle détermine, via $\sim \mathcal{U}$, dans ${ }^{*} \mathbb{R}$. En fait, nous remarquons qu'il existe $j_{0} \in \mathbb{N}$ tel que $O_{\varphi}(j \mid \alpha)=+\infty$ pour $j \geqslant j_{0}$ si et seulement si $\alpha$ est algébrique, et que cette propriété ne dépend pas de la fonction $\varphi$ choisie. Ceci dit nous écrivons $O_{\varphi}(\alpha)=+\infty$ pour tout $\varphi$ lorsque $\tilde{\alpha}=0$, et nous notons $O_{\varphi}(\alpha) \in\left({ }^{*} \mathbb{R}\right)_{+}^{*}$ l'hyperréel déterminé par $O_{\varphi}(\cdot \mid \alpha)$ dès que $\tilde{\alpha} \neq 0$ dans $\mathbb{C} / \sim$.

Remarquons d'une part que si $\varphi \leqslant \varphi^{\prime}$ alors $O_{\varphi}(\alpha) \leqslant O_{\varphi^{\prime}}(\alpha)$ pour tout $\alpha \in \mathbb{C}$, et d'autre part que $O_{\varphi}(\alpha)$ ne dépend en fait que de la classe de $\varphi$ modulo $\sim u$. On peut considérer l'indice $\varphi$ dans la notation $O_{\varphi}(\alpha)$ comme désignant l'hyperréel associé à la fonction $\varphi$, plutôt que la fonction $\varphi$ elle-même.

Lemme 2. Soit $\alpha \in \mathbb{C}$ et $\varphi, \varphi^{\prime} \in \mathcal{C}$ telles que $\varphi \leqslant \varphi^{\prime}$. Alors

(i) $s\left(O_{\varphi}(\alpha)\right) \geqslant s(\varphi)$;

(ii) $O_{\varphi}(\alpha)=O_{\varphi^{\prime}}(\alpha)$ ou $s\left(O_{\varphi}(\alpha)\right) \leqslant s\left(\varphi^{\prime}\right)$.

DÉmonstration: (i) Soient $\alpha \in \mathbb{C}, j \in \mathbb{N}$, le principe des tiroirs montre qu'il existe $P \in \mathbb{Z}[X] \backslash\{0\}$ tel que $\log \|P(\alpha)\| \leqslant-c_{2} \cdot \exp (2 \varphi(j))$ et $\log t(P) \leqslant \varphi(j)$ avec $c_{2}=c_{2}(\alpha) \in \mathbb{R}_{+}^{*}$. D'où $O_{\varphi}(j \mid \alpha) \geqslant \varphi(j)+\log c_{2}$, car $P$ est nécessairement non constant, et donc $s\left(O_{\varphi}(\alpha)\right) \geqslant s(\varphi)$.

(ii) Si $\alpha$ est algébrique alors $O_{\varphi}(\alpha)=O_{\varphi^{\prime}}(\alpha)=+\infty$, sinon on a $O_{\varphi}(\alpha) \leqslant O_{\varphi^{\prime}}(\alpha)$ et posons

$$
E=\left\{j \in \mathbb{N} ; O_{\varphi}(j \mid \alpha)=O_{\varphi^{\prime}}(j \mid \alpha)\right\} .
$$

Si $E \in \mathcal{U}$ alors $O_{\varphi}(\alpha)=O_{\varphi^{\prime}}(\alpha)$, sinon le complémentaire $\bar{E}$ de $E$ dans $\mathbb{N}$ appartient à $\mathcal{U}$. Soient $j \in \bar{E}$ et $P, Q \in \mathbb{Z}[X]$ des polynômes irréductibles, réalisant les maximums des définitions de $O_{\varphi}(j \mid \alpha)$ et $O_{\varphi^{\prime}}(j \mid \alpha)$ respectivement. Comme 
$O_{\varphi}(j \mid \alpha)<O_{\varphi^{\prime}}(j \mid \alpha)$, on a $P \neq Q$ et

$$
\begin{gathered}
0<\left(\log \|P(\alpha)\|^{-1}\right) / t(P)<\left(\log \|Q(\alpha)\|^{-1}\right) / t(Q) \\
\varphi^{\prime}(j) \geqslant \log t(Q)>\varphi(j) \geqslant \log t(P) .
\end{gathered}
$$

Le résultant $r$ de $\mathrm{P}$ et $Q$ est un entier non nul satisfisant

$$
0 \leqslant \log |r| \leqslant \log \|P(\alpha)\|+c_{1} . t(P) \cdot t(Q)
$$

où $c_{1}=c_{1}(\alpha) \in \mathbb{R}_{+}^{*}$, car $\log \|Q(\alpha)\| \leqslant \log \|P(\alpha)\|$. On en déduit

$$
O_{\varphi}(j \mid \alpha)=\log \left(\left(\log \|P(\alpha)\|^{-1}\right) / t(P)\right) \leqslant \log c_{1}+\log t(Q) \leqslant \log c_{1}+\varphi^{\prime}(j) .
$$

On a donc, si $E \notin \mathcal{U}, O_{\varphi}(j \mid \alpha) \leqslant \log c_{1}+\varphi^{\prime}(j)$ pour $j \in \bar{E}$, ce qui entraine $O_{\varphi}(\alpha) / \varphi^{\prime}$ limité et $s\left(O_{\varphi}(\alpha)\right) \leqslant s\left(\varphi^{\prime}\right)$.

Le Lemme 2 permet de contrôler le comportement de $s\left(O_{\varphi}(\alpha) / \varphi\right)$ lorsqu'on fait varier $\varphi$ dans $\mathcal{C}$, on a par exemple:

Corollaire. Soient $\alpha \in \mathbb{C}$ et $\varphi, \varphi^{\prime} \in \mathcal{C}$, $\operatorname{si} \min \left\{s\left(O_{\varphi}(\alpha)\right) ; s\left(O_{\varphi^{\prime}}(\alpha)\right)\right\}>s(\varphi)$ et $s(\varphi)=s\left(\varphi^{\prime}\right)$ alors $O_{\varphi}(\alpha)=O_{\varphi^{\prime}}(\alpha)$. Si $O_{\varphi}(\alpha) / \varphi$ est infiniment grand alors $O_{x \varphi}(\alpha)=O_{\varphi}(\alpha)$ pour tout $x \in \mathbb{R}, x \geqslant 1$. En tout cas il existe $x \in \mathbb{R}, x \geqslant 1$ tel que $O_{y \varphi}(\alpha) / O_{x \varphi}(\alpha)$ soit limité pour tout $y \in \mathbb{R}, y \geqslant 1$.

DÉmonstration: Supposons par exemple $\varphi \leqslant \varphi^{\prime}$. D'après le Lemme 2(ii), si $O_{\varphi}(\alpha)<O_{\varphi^{\prime}}(\alpha)$ on a $s\left(O_{\varphi}(\alpha)\right) \leqslant s\left(\varphi^{\prime}\right)=s(\varphi)$. On applique ensuite le Lemme 2(ii) avec $\varphi^{\prime}=x \varphi$ et, comme $O_{\varphi}(\alpha) / \varphi$ est infiniment grand, on ne peut avoir $s\left(O_{\varphi}(\alpha)\right) \leqslant$ $s\left(\varphi^{\prime}\right)$. Enfin, s'il existe $x \in \mathbb{R}, x \geqslant 1$ tel que $O_{x \varphi}(\alpha) / \varphi$ soit infiniment grand on a, d'après ce qui précède, $O_{y \varphi}(\alpha)=O_{x \varphi}(\alpha)$ pour tout $y \in \mathbb{R}, y \geqslant x$, et donc $O_{y \varphi}(\alpha) \leqslant$ $O_{x \varphi}(\alpha)$ pour tout $y \in \mathbb{R}, y \geqslant 1$. Sinon on a $s\left(O_{\varphi}(\alpha)\right) \geqslant s(\varphi)$ et $O_{y \varphi}(\alpha) / \varphi$ est limité pour tout $y \in \mathbb{R}, y \geqslant 1$, on prend $x=1$ et on vérifie que $s\left(O_{y \varphi}(\alpha) / O_{\varphi}(\alpha)\right) \leqslant$ $s\left(O_{y \varphi}(\alpha) / \varphi\right)$ est limité pour tout $y \in \mathbb{R}, y \geqslant 1$.

REMARque 3. On pourrait de cette facon définir l'ordre de transcendance de $\alpha$ par rapport à $\varphi \in \mathcal{C}$ comme 2 si $s\left(O_{\varphi^{\prime}}(\alpha)\right)=s(\varphi)$ pour toute $\varphi^{\prime} \in \mathcal{C}$ telle que $s\left(\varphi^{\prime}\right)=s(\varphi)$, et $1+s\left(O_{\varphi^{\prime}}(\alpha) / \varphi^{\prime}\right)$ si $\varphi^{\prime} \in \mathcal{C}, s\left(\varphi^{\prime}\right)=s(\varphi)$ et $s\left(O_{\varphi^{\prime}}(\alpha)\right)>s(\varphi)$. On obtient ainsi une notion qui ne dépend pas du choix de $\varphi$ dans sa classe modulo $1+\mathcal{M}$, mais peut beaucoup varier si on multiplie $\varphi$ par 2 par exemple. Afin de pallier ce défaut nous allons sophistiquer notre définition d'ordre de transcendance. La principale difficulté technique de notre démarche est qu'un sous-ensemble borné de ' $\mathbb{R}$ n'a pas nécessairement de borne supérieure dans * $\overline{\mathbb{R}}$, c'est là qu'intervient le Lemme 1 . 
REMARQUE 4. L'ordre de transcendance décrit dans la Remarque 3 conduit à une classification des nombres complexes proche de celle de [8], mais néanmoins plus grossière. Cette classification a toutefois l'avantage que deux nombres $y$ sont toujours comparables. L'ordre de transcendance que nous allons introduire ci-après conduit, lui, à une classification moins fine que les précédentes.

Soit $\alpha \in \mathbb{C}$, le Lemme 2 montre que, si $\tilde{\alpha} \neq 0$, la fonction $f$ définie par $f(a)=: s\left(O_{a \varphi}(\alpha) / \varphi\right)$ si $a \in\left({ }^{*}\right)_{+}^{*}, a \geqslant 1$ (et $f(a)=1$ pour $a<1$, par exemple) satisfait les hypothèses du Lemme 1 avec $\ell=c=1$. Les ensembles $E_{j}(f)(f \in \mathbb{N})$ admettent donc des bornes supérieures dans $\Gamma_{1}$ et la suite $\left(\sup E_{j}(f) / 2^{j}\right)_{j \in N}$ définit bien un dihyperréel $a(f) \in \Gamma$. Nous noterons $\rho_{\varphi}(\alpha)=a(f)+1$ si $\tilde{\alpha} \neq 0$ et poserons $\rho_{\varphi}(\alpha)=+\infty$ si $\bar{\alpha}=0$.

DÉfinition: Pour $\alpha \in \mathbb{C}, \varphi \in \mathcal{C}$, on appelera ordre de transcendance (relatif $\dot{a}$ $\varphi(\mathcal{U})$ de $\alpha$, l'élément $\rho_{\varphi}(\alpha)$ de $\bar{\Gamma}$ défini ci-dessus.

NOTA BENE: Cette notion d'ordre de transcendance diffère sensiblement des notions habituellement utilisées aussi appelées "types de transcendance" (voir Remarque 9 à la fin du Section 4). On remarquera, par exemple, que notre notion dépend aussi du choix de l'ultrafiltre $\mathcal{U}$ fixé. Nous n'avons pas fait figurer cette dépendance dans la notation $\rho_{\varphi}(\alpha)$ et on pourra aussi oublier l'indice $\varphi$ lorsqu'il n'y aura pas risque de confusion.

Proposition 1. Soient $\alpha, \alpha^{\prime} \in \mathbb{C}, \varphi, \varphi^{\prime} \in \mathcal{C}, x \in \mathbb{R}, x \geqslant 1$ et $a \in \Gamma_{1}^{*}$. On a:

(o) $\rho_{\varphi}(\alpha) \geqslant 2$;

(i) s'il existe $y \in \mathbb{R}, y \geqslant 1$ tel que $O_{y \varphi}(\alpha) / \varphi \notin \mathcal{L}$, alors

$$
\rho_{x \varphi}(\alpha)-1 \leqslant(1 / x) \cdot\left(\rho_{\varphi}(\alpha)-1\right)
$$

(ii) si $s\left(O_{b \varphi}(\alpha) / \varphi\right) \leqslant a . s\left(O_{b \varphi^{\prime}}\left(\alpha^{\prime}\right) / \varphi^{\prime}\right)$ pour $b \in * \mathbb{R}, b \geqslant 1$ alors

$$
\rho_{\varphi}(\alpha)-1 \leqslant a \cdot\left(\rho_{\varphi^{\prime}}\left(\alpha^{\prime}\right)-1\right)
$$

(iii) si $s(\varphi)=s\left(\varphi^{\prime}\right)$ alors $\rho_{\varphi}(\alpha)=\rho_{\varphi^{\prime}}(\alpha)$;

(iv) l'ordre de transcendance $\rho_{\varphi}(\alpha) \in \bar{\Gamma}$ ne dépend que de la classe de $\alpha$ dans $\mathbb{C} / \sim$, et ne dépend pas du choix de la taille $t$ dans $\mathcal{T}$.

DÉmonstration: (o) On a, d'après le Lemme 2(i), $s\left(O_{b \varphi}(\alpha) / b \varphi\right) \geqslant 1$ pour tout $b \in \mathbb{R}, b \geqslant 1$, et donc, par définition, $\rho_{\varphi}(\alpha) \geqslant a(f)+1 \geqslant 2$ avec $f(b)=: s\left(O_{b \varphi}(\alpha) / \varphi\right)$.

(i) On a $\rho_{\varphi}(\alpha)-1=a(f)$ et $\rho_{x \varphi}(\alpha)-1=a\left(f^{\prime}\right)$ où $f$ et $f^{\prime}$ sont définies par $f(b)=s\left(O_{b \varphi}(\alpha) / \varphi\right)$ et $f^{\prime}(b)=s\left(O_{b x \varphi}(\alpha) / x \varphi\right)$. Soit $n \in \mathbb{N}$ tel que $2^{n} \geqslant x$, on a $a\left(f^{\prime}\right) \leqslant(1 / x) \cdot a_{n}(f)$, d'où le résultat avec le Lemme 1 car il existe $y \in \mathbb{R}, y \geqslant 1$ tel que $f(y) \notin s(\mathcal{L})$. 
(ii) Soit $f(b)=s\left(O_{b \varphi}(\alpha) / \varphi\right)$ et $f^{\prime}(b)=s\left(O_{b \varphi^{\prime}}\left(\alpha^{\prime}\right) / \varphi^{\prime}\right)$, comme $f \leqslant a f^{\prime}$ par hypothèse on a, par définition, $a(f) \leqslant a . a\left(f^{\prime}\right)$, c'est-à-dire $\rho_{\varphi}(\alpha)-1 \leqslant a .\left(\rho_{\varphi^{\prime}}\left(\alpha^{\prime}\right)-1\right)$.

(iii) Avec les notations ci-dessus, si $s(\varphi)=s\left(\varphi^{\prime}\right)$ et $\alpha=\alpha^{\prime}$, les ensembles $E_{j}(f)$ et $E_{j}\left(f^{\prime}\right)$ coincident, on a donc, encore par définition, $\rho_{\varphi}(\alpha)=\rho_{\varphi^{\prime}}(\alpha)$.

(iv) Si $\alpha$ et $\alpha^{\prime}$ sont dans la même classe de $\mathbb{C} / \sim$ il existe $Q \in \mathbb{Z}\left[X, X^{\prime}\right] \backslash\{0\}$, irréducible tel que $Q\left(\alpha, \alpha^{\prime}\right)=0$. Soient $j \in \mathbb{N}, b \in{ }^{*} \mathbb{R}, b \geqslant 1$ et $P \in \mathbb{Z}[X] \backslash \mathbb{Z}$ réalisant le maximum dans la définition de $O_{b \varphi}(j \mid \alpha)$ et satisfaisant $b \varphi(j)-\log 2 \leqslant$ $\log t(P) \leqslant b \varphi(j)$, prenant le résultant par rapport à $X$ de $P$ et $Q$ on obtient un polynôme $P^{\prime} \in \mathbb{Z}\left[X^{\prime}\right] \backslash \mathbb{Z}$ satisfaisant

$$
\log t\left(P^{\prime}\right) \leqslant b \varphi(j)+c_{1} \quad \text { et } \quad \log \left\|P^{\prime}\left(\alpha^{\prime}\right)\right\| \leqslant \log \|P(\alpha)\|+c_{2} . t(P)
$$

où $c_{1}, c_{2}$ sont des réels $\geqslant 1$ ne dépendant pas de $a$ et $j$. On en déduit, pour $j \in \mathbb{N}$ assez grand,

$$
O_{b \varphi+c_{1}}\left(j \mid \alpha^{\prime}\right) \geqslant O_{b \varphi}(j \mid \alpha)-c_{3}
$$

car $\log \|P(\alpha)\| \leqslant-c_{3} \cdot t(P) \cdot \varphi(j) \leqslant-2 c_{2} . t(P)$. D'où avec le Lemme 2(i) $s\left(O_{b\left(\varphi+c_{1}\right)}\left(\alpha^{\prime}\right)\right) \geqslant s\left(O_{b \varphi}(\alpha)\right)$ pour tout $b \in \mathbb{R}, b \geqslant 1$. On montre une inégalité inverse de la même facon, et ensuite avec (ii), (iii) et $s\left(\varphi+c_{1}\right)=s(\varphi)$ on obtient

$$
\rho_{\varphi}(\alpha) \leqslant \rho_{\varphi+c_{1}}\left(\alpha^{\prime}\right)=\rho_{\varphi}\left(\alpha^{\prime}\right) \leqslant \rho_{\varphi+c_{1}}(\alpha)=\rho_{\varphi}(\alpha) .
$$

Si on remplace la taille $t$ par une taille $t^{\prime}$ satisfaisant

$$
c^{-1} . t \leqslant t^{\prime} \leqslant c . t
$$

avec $c \in \mathbb{R}, c \geqslant 1$, on vérifie sans peine, pour tout $b \in{ }^{*} \mathbb{R}, b \geqslant 1, O_{b \varphi}(j \mid \alpha) \leqslant$ $O_{b(\varphi+\log c)}^{\prime}(j \mid \alpha)+\log c$ et $O_{b \varphi}^{\prime}(j \mid \alpha) \leqslant O_{b(\varphi+\log c)}(j \mid \alpha)+\log c$. On en déduit $\rho_{\varphi}(\alpha) \leqslant$ $\rho_{\varphi}^{\prime}(\alpha)$ et $\rho_{\varphi}^{\prime}(\alpha) \leqslant \rho_{\varphi}(\alpha)$ comme précédemment avec (ii), (iii) et $s(\varphi+\log c)=s(\varphi)$.

Remarque 5. L'ordre de transcendance de $\alpha \in \mathbb{C}$ ne dépendant que de la classe $\widetilde{\alpha}$ de $\alpha$ dans $\mathbb{C} / \sim$ sera aussi noté $\rho_{\varphi}(\tilde{\alpha})$. Si $u \in \mathbb{C} / \sim$ on aura $\rho_{\varphi}(u)=\rho_{\varphi}(\alpha)$ pour tout $\alpha \in \mathbb{C}$ tel que $\tilde{\alpha}=u$.

(B) Approximation MUtuelle. On associe maintenant à chaque application $O_{\varphi}(\cdot \mid \alpha, \beta)$ l'hyperréel qu'elle détermine, via $\sim \mathcal{U}$, dans ${ }^{*} \mathbb{R}$. Comme en $(\mathrm{A})$, nous remarquons qu'il existe $j_{0} \in \mathbb{N}$ tel que $O_{\varphi}(j \mid \alpha, \beta)=+\infty$ pour $j \geqslant j_{0}$ si et seulement si $\alpha$ et $\beta$ dépendent algébriquement l'un de l'autre, et que cette propriété ne dépend pas de la fonction $\varphi$ choisie. Ceci dit nous écrivons $O_{\varphi}(\alpha, \beta)=+\infty$ pour tout $\varphi$ lorsque $\tilde{\alpha}=\widetilde{\beta}$, et nous notons $O_{\varphi}(\alpha, \beta) \in\left({ }^{*} \mathbb{R}\right)_{+}^{*}$ l'hyperréel déterminé par $O_{\varphi}(\cdot \mid \alpha, \beta)$ dès que $\tilde{\alpha} \neq \tilde{\beta}$ dans $\mathbb{C} / \sim$.

Remarquons à nouveau que si $\varphi \leqslant \varphi^{\prime}$ alors $O_{\varphi}(\alpha, \beta) \leqslant O_{\varphi^{\prime}}(\alpha, \beta)$ pour tout $\alpha, \beta \in \mathbb{C}$, et que $O_{\varphi}(\alpha, \beta)$ ne dépend que de l'hyperréel déterminé par la fonction $\varphi$. 
Lemme 3. Soient $\alpha, \beta \in \mathbb{C}$ et $\varphi, \varphi^{\prime}, \varphi^{\prime \prime} \in \mathcal{C}$ telles que $\varphi \leqslant \varphi^{\prime} \leqslant \varphi^{\prime \prime}$. Alors

(i) $s\left(O_{\varphi+2 \log 2}(\alpha, \beta)\right) \geqslant s\left(\min \left\{O_{\varphi}(\alpha) ; O_{\varphi}(\beta)\right\}\right) \geqslant s(\varphi)$;

(ii) $O_{\varphi^{\prime \prime}}(\alpha, \beta)=O_{\varphi^{\prime}}(\alpha, \beta)$ ou $s\left(O_{\varphi^{\prime}}(\alpha, \beta)\right) \leqslant s\left(O_{\varphi}(\alpha, \beta)+\varphi+\varphi^{\prime \prime}\right)$ ou $s\left(O_{\varphi}(\alpha, \beta)\right) \leqslant s\left(\varphi^{\prime}+\varphi^{\prime \prime}\right)$.

DÉmonstration: (i) Soient $\alpha, \beta \in \mathbb{C}$, si $P$ et $Q$ sont des polynômes réalisant les maximums dans les définitions de $O_{\varphi}(j \mid \alpha)$ et $O_{\varphi}(j \mid \beta)$ respectivement, satisfaisant $\varphi(j) \geqslant \log t(P), \log t(Q) \geqslant \varphi(j)-\log 2$, alors le polynôme $P+Q \in \mathbb{Z}[X, Y]$ est sans facteur dans $\mathbb{Z}[X] \cup \mathbb{Z}[Y]$ (car $P$ et $Q$ sont non constants) et satisfait

$$
\begin{aligned}
& \log t(P+Q) \leqslant \max \{\log t(P) ; \log t(Q)\}+2 \cdot \log 2 \leqslant \varphi(j)+2 \cdot \log 2, \\
& \log \|(P+Q)(\alpha, \beta)\| \leqslant \max \{\log \|P(\alpha)\| ; \log \|Q(\beta)\|\}+c_{1} \cdot \exp (\varphi(j)) .
\end{aligned}
$$

D'où

$$
\frac{\log \|(P+Q)(\alpha, \beta)\|^{-1}}{t(P+Q)} \geqslant \frac{1}{8} \cdot \min \left\{\frac{\log \|P(\alpha)\|^{-1}}{t(P)} ; \frac{\log \|Q(\beta)\|^{-1}}{t(Q)}\right\}-c_{2},
$$

par suite $O_{\varphi+2 \log 2}(\alpha, \beta) \geqslant \min \left\{O_{\varphi}(\alpha) ; O_{\varphi}(\beta)\right\} .(1+\epsilon)$ avec $\epsilon \in \mathcal{M}$. Et on complète la démonstration de (i) avec le Lemme $2(\mathrm{i})$.

(ii) Si $\alpha$ et $\beta$ sont algébriquement dépendants alors $O_{\varphi}(\alpha, \beta)=O_{\varphi^{\prime}}(\alpha, \beta)=$ $O_{\varphi^{\prime \prime}}(\alpha, \beta)=+\infty$, sinon on a $O_{\varphi}(\alpha, \beta) \leqslant O_{\varphi^{\prime}}(\alpha, \beta) \leqslant O_{\varphi^{\prime \prime}}(\alpha, \beta)$ et nous considérons l'ensemble

$$
\begin{aligned}
E=\left\{j \in \mathbb{N} ; O_{\varphi^{\prime \prime}}(j \mid \alpha, \beta) \leqslant O_{\varphi^{\prime}}(j \mid \alpha, \beta)\right. & \\
& \text { ou } \left.O_{\varphi^{\prime}}(j \mid \alpha, \beta) \leqslant O_{\varphi}(j \mid \alpha, \beta)+\varphi(j)+\varphi^{\prime \prime}(j)+c\right\}
\end{aligned}
$$

où $c$ est un réel $>0$ indépendant de $j$ qui pourrait-être explicité. Si $E \in \mathcal{U}$ alors $O_{\varphi^{\prime \prime}}(\alpha, \beta)=O_{\varphi^{\prime}}(\alpha, \beta)$ ou $s\left(O_{\varphi^{\prime}}(\alpha, \beta)\right) \leqslant s\left(O_{\varphi}(\alpha, \beta)+\varphi+\varphi^{\prime \prime}\right)$, sinon $\bar{E} \in$ $\mathcal{U}$. Soient $j \in \bar{E}$ et $Q, R \in \mathbb{Z}[X, Y]$ des polynômes irréducibles réalisant les maximums des définitions de $O_{\varphi^{\prime}}(j \mid \alpha, \beta)$ et $O_{\varphi^{\prime \prime}}(j \mid \alpha, \beta)$ respectivement. Comme $O_{\varphi^{\prime}}(j \mid \alpha, \beta)<O_{\varphi^{\prime \prime}}(j \mid \alpha, \beta)$ on a $Q \neq R$ et l'idéal $I=(Q, R)$ de $\mathbb{Z}[X, Y]$ est de rang 2. Avec [9] et en homogénéisant, on vérifie que $I$ est de taille $t(I) \leqslant c_{1} . t(Q) . t(R) \leqslant$ $c_{1} \cdot \exp \left(\varphi^{\prime}(j)+\varphi^{\prime \prime}(j)\right)$ et satisfait

$$
\log \|I(\alpha, \beta)\| \leqslant \log \|Q(\alpha, \beta)\|+c_{2} . t(Q) . t(R),
$$

car on montre comme dans la preuve du Lemme $2(\mathrm{i}) \log \|R(\alpha, \beta)\| \leqslant \log \|Q(\alpha, \beta)\|$ (On a noté ici $\|I(\alpha, \beta)\|$ plutôt que $\|I\|_{(\alpha, \beta)}$ comme dans [9]). Pour $j \in \bar{E}$ on a

$$
-\exp \left(O_{\varphi^{\prime}}(j \mid \alpha, \beta)\right)=\frac{\log \|Q(\alpha, \beta)\|}{t(Q)}<-c_{2} \cdot \exp \left(\varphi^{\prime \prime}(j)\right) \leqslant-c_{2} \cdot t(R),
$$

d'où

$$
\frac{\log \|I(\alpha, \beta)\|}{t(I)} \leqslant \frac{\log \|Q(\alpha, \beta)\|}{c_{1} . t(Q) t(R)}+\frac{c_{2}}{c_{1}}
$$


On déduit alors des propriétés d'additivité et de multiplicativité de $t(I)$ et $\|I(\alpha, \beta)\|$ l'existence d'un idéal premier $\mathfrak{p}$ de $\mathbb{Z}[X, Y]$ associé à $I$, de rang 2 , de taille $t(\mathfrak{p}) \leqslant$ $c_{1} . t(Q) \cdot t(R)$ et satisfaisant

$$
\frac{\log \|\mathfrak{p}(\alpha, \beta)\|}{t(\mathfrak{p})} \leqslant \frac{\log \|Q(\alpha, \beta)\|}{c_{1} . t(Q) t(R)}+\frac{c_{2}}{c_{1}}=-\left(c_{1} . t(R)\right)^{-1} \cdot \exp \left(O_{\varphi^{\prime}}(j \mid \alpha, \beta)\right)+\left(c_{2} / c_{1}\right) .
$$

Il suit du Lemme 2.7 de [9] que $\mathfrak{p}$ a un zéro dans une boule de $\mathbb{C}^{2}$ de centre $(\alpha, \beta)$ et de rayon $c_{3} \cdot \exp \left(-\left(c_{1} . t(R)\right)^{-1} \cdot \exp \left(O_{\varphi^{\prime}}(j \mid \alpha, \beta)\right)\right)$. Soit maintenant $P \in \mathbb{Z}[X, Y]$ réalisant le maximum de $O_{\varphi}(j \mid \alpha, \beta)$, si $P \in \mathfrak{p}$ alors $P$ s'annule au zéro de $\mathfrak{p}$ ci-dessus et par continuité

$$
\exp \left(O_{\varphi}(j \mid \alpha, \beta)\right)=\frac{\log \left(\|P(\alpha, \beta)\|^{-1}\right)}{t(P)} \geqslant \frac{\exp \left(O_{\varphi^{\prime}}(j \mid \alpha, \beta)\right)}{c_{1} \cdot t(P) \cdot t(R)}-c_{4},
$$

d'où $O_{\varphi^{\prime}}(j \mid \alpha, \beta) \leqslant O_{\varphi}(j \mid \alpha, \beta)+\varphi(j)+\varphi^{\prime \prime}(j)+c$ en contradiction avec $j \in \bar{E}$. Ainsi $P \notin \mathfrak{p}$ et l'idéal $(\mathfrak{p}, P)$ est de rang 3 , et tous ses premiers associés intersectent $\mathbb{Z}$. On a $t((\mathfrak{p}, P)) \leqslant c_{1} \cdot t(\mathfrak{p}) \cdot t(P)$ et

$$
\begin{aligned}
-c_{8} . t((\mathfrak{p}, P)) & \leqslant \log \|(\mathfrak{p}, P)(\alpha, \beta)\| \\
& \leqslant c_{7} \cdot \max \{\log \|\mathfrak{p}(\alpha, \beta)\| ; \log \|P(\alpha, \beta)\|\}+c_{8} . t(\mathfrak{p}) \cdot t(P),
\end{aligned}
$$

On en déduit

$$
\exp \left(O_{\varphi^{\prime}}(j \mid \alpha, \beta)\right) \leqslant c_{1} \cdot t(R) \cdot \frac{\log \left(\|\mathfrak{p}(\alpha, \beta)\|^{-1}\right)}{t(\mathfrak{p})}+c_{2} \cdot t(R) \leqslant c_{10} \cdot \exp \left(\varphi(j)+\varphi^{\prime \prime}(j)\right)
$$
ou

$$
\exp \left(O_{\varphi}(j \mid \alpha, \beta)\right)=\frac{\log \left(\|P(\alpha, \beta)\|^{-1}\right)}{t(P)} \leqslant c_{0} . t(p) \leqslant c_{10} \cdot \exp \left(\varphi^{\prime}(j)+\varphi^{\prime \prime}(j)\right)
$$

pour tout $j \in \bar{E}$. La première éventualité est exclue lorsque $j \in \bar{E}$, et donc si $\bar{E} \in \mathcal{U}$ on a $s\left(O_{\varphi}(\alpha, \beta)\right) \leqslant s\left(\varphi^{\prime}+\varphi^{\prime \prime}\right)$, ce qui achève de montrer (ii).

Soient $\alpha, \beta \in \mathbb{C}$, le Lemme 3 montre que, si $\tilde{\alpha} \neq \tilde{\beta}$, la fonction $f$ définie par $f(a)=: s\left(O_{a \varphi}(\alpha, \beta) / \varphi\right)$ si $a \in(\mathbb{R})_{+}^{*}, a \geqslant 1$ (et $f(a)=1$ si $a<1$ ) satisfait les hypothèses du Lemme 1 avec $\ell=c=2$. Les ensembles $E_{j}(f)(j \in \mathbb{N})$ admettent donc des bornes supérieures dans $\Gamma_{1}$ et la suite $\left(\sup E_{j}(f) / 2^{j}\right)_{j \in \mathbb{N}}$ définit bien un dihyperréel $a(f) \in \Gamma$. Nous noterons $\rho_{\varphi}(\alpha, \beta)=a(f)+1$ si $\tilde{\alpha} \neq \tilde{\beta}$ et poserons $\rho_{\varphi}(\alpha, \beta)=+\infty$ si $\tilde{\boldsymbol{\alpha}}=\tilde{\boldsymbol{\beta}}$.

DÉfINITION: Pour $\alpha, \beta \in \mathbb{C}, \varphi \in \mathcal{C}$, on appelera ordre d'approximation mutuelle (relatif à $\varphi(\mathcal{U})$ ) de $(\alpha, \beta)$, l'élément $\rho_{\varphi}(\alpha, \beta)$ de $\bar{\Gamma}$ défini ci-dessus. 
Remarque 6. On a $\rho_{\varphi}(\alpha, \beta)=+\infty$ si et seulement s'il existe $x \in \mathbb{R}, x \geqslant 1$ tel que $O_{x \varphi}(\alpha, \beta)=+\infty$, i.e. si et seulement si $\alpha$ et $\beta$ dépendent algébriquement l'un de l'autre (i.e. $\widetilde{\alpha}=\widetilde{\beta}$ ). De même on a $\rho_{\varphi}(\alpha)=+\infty$ si et seulement si $\alpha$ est algébrique sur $\mathbb{Q}$ (i.e. $\tilde{\alpha}=0$ ).

REMARQUe 7. Comme pour l'ordre de transcendance, notre notion d'ordre d'approximation dépend du choix de l'ultrafiltre $\mathcal{U}$ fixé et nous omettrons l'indice $\varphi$ le cas échéant.

Proposition 2. Soient $\alpha, \beta, \alpha^{\prime}, \beta^{\prime} \in \mathbb{C}, \varphi, \varphi^{\prime} \in \mathcal{C}, x \in \mathbb{R}, x \geqslant 1$ et $a \in \Gamma_{1}^{*}$. On a:

(o) $\rho_{\varphi}(\alpha, \beta) \geqslant 2$;

(i) s'il existe $y \in \mathbb{R}, y \geqslant 1$ tel que $O_{y \varphi}(\alpha, \beta) / \varphi \notin \mathcal{L}$ alors

$$
\rho_{x \varphi}(\alpha, \beta)-1 \leqslant(1 / x) \cdot\left(\rho_{\varphi}(\alpha, \beta)-1\right) ;
$$

(ii) si $s\left(O_{b \varphi}(\alpha, \beta) / \varphi\right) \leqslant a . s\left(O_{b \varphi^{\prime}}\left(\alpha^{\prime}, \beta^{\prime}\right) / \varphi^{\prime}\right)$ pour $b \in \mathbb{R}^{*}, b \geqslant 1$ alors

$$
\rho_{\varphi}(\alpha, \beta)-1 \leqslant a .\left(\rho_{\varphi^{\prime}}\left(\alpha^{\prime}, \beta^{\prime}\right)-1\right) ;
$$

(iii) si $s(\varphi)=s\left(\varphi^{\prime}\right)$ alors $\rho_{\varphi}(\alpha, \beta)=\rho_{\varphi^{\prime}}(\alpha, \beta)$;

(iv) $\rho_{\varphi}(\alpha, \beta)=\rho_{\varphi}(\beta, \alpha), \rho_{\varphi}(\alpha, 0)=\rho_{\varphi}(\alpha)$ et $\rho_{\varphi}(\alpha, \beta)=+\infty$ si et seulement si $\tilde{\alpha}=\widetilde{\beta}$

(v) l'ordre d'approximation mutuelle $\rho_{\varphi}(\alpha, \beta) \in \bar{\Gamma}$ ne dépend que des classes de $\alpha$ et $\beta$ dans $\mathbb{C} / \sim$, et ne dépend pas du choix de la taille $t$ dans $\mathcal{T}$.

DÉmonstration: Elle recopie celle de la Proposition 1.

(o) On a clairement $\rho_{\varphi}(\alpha, \beta) \geqslant 2$ par définition, avec le Lemme 3(i).

(i) On a $\rho_{\varphi}(\alpha, \beta)-1=a(f)$ et $\rho_{x \varphi}(\alpha, \beta)-1=a\left(f^{\prime}\right)$ où $f$ et $f^{\prime}$ sont définies par $f(b)=s\left(O_{b \varphi}(\alpha, \beta) / \varphi\right)$ et $f^{\prime}(b)=s\left(O_{b x \varphi}(\alpha, \beta) / x \varphi\right)$. Soit $n \in \mathbb{N}$ tel que $2^{n} \geqslant x$, on a $a\left(f^{\prime}\right) \leqslant(1 / x) \cdot a_{n}(f)$, d'où le résultat avec le Lemme 1 car il existe $y \in \mathbb{R}, y \geqslant 1$ tel que $f(y) \notin s(\mathcal{L})$.

(ii) Soient $f(b)=s\left(O_{b \varphi}(\alpha, \beta) / \varphi\right)$ et $f^{\prime}(b)=s\left(O_{b \varphi^{\prime}}\left(\alpha^{\prime}, \beta^{\prime}\right) / \varphi^{\prime}\right)$, comme $f \leqslant$ $a f^{\prime}$ par hypothèse on a, par définition, $a(f) \leqslant a . a\left(f^{\prime}\right)$, c'est-à-dire $\rho_{\varphi}(\alpha, \beta)-1=$ a. $\left(\rho_{\varphi^{\prime}}\left(\alpha^{\prime}, \beta^{\prime}\right)-1\right)$.

(iii) A vec les notations ci-dessus, si $s(\varphi)=s\left(\varphi^{\prime}\right)$ et $\alpha=\alpha^{\prime}, \beta=\beta^{\prime}$, les ensembles $E_{j}(f)$ et $E_{j}\left(f^{\prime}\right)$ coinncident, on a donc encore par définition $\rho_{\varphi}(\alpha, \beta)=\rho_{\varphi^{\prime}}(\alpha, \beta)$.

(iv) Pour $b \in \mathbb{R}^{\mathbb{R}}, b \geqslant 1$ on vérifie directement sur les définitions (et avec le Lemme 1.13 de [9], par exemple) $O_{b \varphi}(\alpha, \beta)=O_{b \varphi}(\beta, \alpha)$ et

$$
O_{b \varphi}(\alpha, 0) \leqslant O_{b \varphi}(\alpha) \leqslant O_{b \varphi+c_{1}}(\alpha, 0)
$$


D'où on déduit $\rho_{\varphi}(\beta, \alpha)-1=\rho_{\varphi}(\alpha, \beta)-1$ et $\rho_{\varphi}(\alpha, 0)-1=\rho_{\varphi}(\alpha)-1$. Par définition $\rho_{\varphi}(\alpha, \beta)=+\infty$ si et seulement s'il existe $y \in \mathbb{R}, y \geqslant 1$ tel que $O_{y \varphi}(\alpha, \beta)=+\infty$, ce qui est bien équivalent à $\widetilde{\alpha}=\widetilde{\beta}$ (voir Remarque 8).

(v) Si $\alpha$ et $\alpha^{\prime}$ sont dans la même classe dans $\mathbb{C} / \sim$ il existe $Q \in \mathbb{Z}\left[X, X^{\prime}\right] \backslash\{0\}$, irréducible tel que $Q\left(\alpha, \alpha^{\prime}\right)=0$. Soit $b \in \mathbb{R}, b \geqslant 1$ et $j \in \mathbb{N}$, prenant le résultant par rapport à $X$ de $Q$ et d'un polynôme $P \in \mathbb{Z}[X, Y]$ réalisant le maximum dans la définition de $O_{b \varphi}(j \mid \alpha, \beta)$ on obtient un polynôme $P^{\prime} \in \mathbb{Z}\left[X^{\prime}, Y\right] \backslash\{0\}$ satisfaisant:

$$
\log t\left(P^{\prime}\right) \leqslant b \varphi(j)+c_{1} \quad \text { et } \quad \log \left\|P^{\prime}\left(\alpha^{\prime}, \beta\right)\right\| \leqslant \log \|P(\alpha, \beta)\|+c_{2} . t(P) .
$$

On en déduit $O_{b\left(\varphi+c_{1}\right)}\left(j \mid \alpha^{\prime}, \beta\right) \geqslant O_{b \varphi}(j \mid \alpha, \beta) \cdot(1+\epsilon(j))$ où $\epsilon \in \mathcal{M}$. Il suit

$$
s\left(O_{b\left(\varphi+c_{1}\right)}\left(\alpha^{\prime}, \beta\right)\right) \geqslant s\left(O_{b \varphi}(\alpha, \beta)\right)
$$

pour tout $b \in{ }^{*} \mathbb{R}, b \geqslant 1$. Et avec (ii), (iii) et $s\left(\varphi+c_{1}\right)=s(\varphi)$ on obtient $\rho_{\varphi}(\alpha, \beta) \leqslant$ $\rho_{\varphi+c_{1}}\left(\alpha^{\prime}, \beta\right)=\rho_{\varphi}\left(\alpha^{\prime}, \beta\right)$. L'inégalité inverse se montre de la même façon.

Si on remplace la taille $t$ par une taille $t^{\prime}$ satisfaisant

$$
c^{-1} . t \leqslant t^{\prime} \leqslant c . t,
$$

pour $c \in \mathbb{R}, c \geqslant 1$ on vérifie, pour $b \in \mathbb{R}, b \geqslant 1$ et $j \in \mathbb{N}$,

$$
O_{b \varphi}(j \mid \alpha, \beta) \leqslant O_{b(\varphi+\log c)}^{\prime}(j \mid \alpha, \beta)+\log c .
$$

On en déduit $\rho_{\varphi}(\alpha, \beta) \leqslant \rho^{\prime}(\alpha, \beta)$ grâce à (ii) et (iii), car $s(\varphi+\log c)=s(\varphi)$. L'inégalité inverse se démontre pareillement.

Remarque 8. Si $\varphi \in \mathcal{C}$, l'ordre d'approximation mutuelle $\rho_{\varphi}(\alpha, \beta)$ de $\alpha$ et $\beta$ est bien défini et ne dépend donc que des classes de $\alpha$ et $\beta$ dans $\mathbb{C} / \sim$, nous le noterons encore $\rho_{\varphi}(\tilde{\alpha}, \tilde{\beta})$. Lorsque $\tilde{\beta}=0$ on a $\rho_{\varphi}(\tilde{\alpha}, 0)=\rho_{\varphi}(\tilde{\alpha})$.

REMARQUE 9. Si $\bar{\rho}(\tilde{\alpha}, \tilde{\beta}) \neq+\infty$ alors $O_{\varphi}(\alpha, \beta) / \varphi$ est limité pour tout $\varphi \in \mathcal{C}$ et

$$
\rho_{\varphi}(\tilde{\alpha}, \tilde{\beta}) \leqslant \bar{\rho}(\tilde{\alpha}, \tilde{\beta}) .
$$

\section{Distance locale et topologie}

Dans tout ce paragraphe nous fixons une fonction $\varphi \in \mathcal{C}$, nous omettrons l'indice correspondant dans les notations $\rho_{\varphi}(\alpha), \rho_{\varphi}(\alpha, \beta)$.

On définit la fonction dam: $(\mathbb{C} / \sim)^{2} \rightarrow \Gamma$ par $\operatorname{dam}(u, u)=0$ et si $u \neq v$,

$$
\operatorname{dam}(u, v)=2 / \rho(u, v) .
$$


ThÉorìme. La fonction dam est bien définie et ne dépend pas du choix de la taille $t$ dans $\mathcal{T}$. Elle induit une distance locale non standard sur $\mathbb{C} / \sim$, c'est-à-dire vérifie, pour tous $u, v, w \in \mathbb{C} / \sim$ :

(i) $\operatorname{dam}(u, v)=0 \Leftrightarrow u=v$

(ii) $\operatorname{dam}(u, v)=\operatorname{dam}(v, u)$

(iii) $\operatorname{dam}(u, w) \leqslant \max \{\operatorname{dam}(u, v) ; \operatorname{dam}(v, w)\}$ dès que $\operatorname{dam}(u, v)$ et $\operatorname{dam}(v, w)$ sont plus petits qu'un infiniment petit de $\Gamma_{1}$.

On a de plus $\operatorname{dam}(u, v) \leqslant 1$.

La démonstration du théorème repose en partie sur le lemme suivant.

Lemme 4. Soient $\alpha, \beta, \gamma \in \mathbb{C}$ et $\varphi, \varphi^{\prime} \in \mathcal{C}$, alors

$$
\begin{aligned}
& s\left(\frac{O_{\varphi+\varphi^{\prime}+\log _{\theta}}(\alpha, \gamma)+\varphi+\varphi^{\prime}}{\varphi+\varphi^{\prime}}\right) \\
& \quad \geqslant s\left(\min \left\{\frac{\varphi}{\varphi+\varphi^{\prime}} \cdot \frac{O_{\varphi}(\alpha, \beta)+\varphi}{\varphi} ; \frac{\varphi^{\prime}}{\varphi+\varphi^{\prime}} \cdot \frac{O_{\varphi^{\prime}}(\beta, \gamma)+\varphi^{\prime}}{\varphi^{\prime}}\right\}\right) .
\end{aligned}
$$

En particulier, s'il existe $y \in \mathbb{R}, y \geqslant 1$ tel que $O_{y \varphi}(\alpha, \beta) / \varphi$ et $O_{y \varphi}(\beta, \gamma) / \varphi \notin \mathcal{L}$ on a $\rho_{\varphi}(\alpha, \gamma) \geqslant \min \left\{\rho_{\varphi}(\alpha, \beta) ; \rho_{\varphi}(\beta, \gamma)\right\}$.

DÉmonstration: Soient $j \in \mathbb{N}$ et $P \in \mathbb{Z}[X, Y], Q \in \mathbb{Z}[Y, Z]$ réalisant les maximums des définitions de $O_{\varphi}(j \mid \alpha, \beta)$ et $O_{\varphi^{\prime}}(j \mid \beta, \gamma)$ respectivement et satisfaisant

$$
\varphi(j)-\log 2 \leqslant \log t(P) \leqslant \varphi(j) \quad \text { et } \quad \varphi^{\prime}(j)-\log 2 \leqslant \log t(Q) \leqslant \varphi^{\prime}(j) .
$$

Prenant le résultant de $P$ et $Q$ par rapport à $Y$ on obtient un polynôme $R \in \mathbb{Z}[X, Z]$, sans facteur dans $\mathbb{Z}[X] \cup \mathbb{Z}[Z]$ et satisfaisant

$$
\begin{gathered}
\log t(R) \leqslant \log t(P)+\log t(Q)+\log 6 \leqslant \varphi(j)+\varphi^{\prime}(j)+\log 6, \\
\log \|R(\alpha, \gamma)\| \leqslant \max \{\log \|P(\alpha, \beta)\| ; \log \|Q(\beta, \gamma)\|\}+c_{1} \cdot \exp \left(\varphi(j)+\varphi^{\prime}(j)\right) .
\end{gathered}
$$

On en déduit

$$
O_{\varphi+\varphi^{\prime}+\log \delta}(j \mid \alpha, \gamma) \geqslant \min \left\{O_{\varphi}(j \mid \alpha, \beta)-\varphi^{\prime}(j) ; O_{\varphi^{\prime}}(j \mid \beta, \gamma)-\varphi(j)\right\}-c_{2} .
$$

I suit, avec le Lemme 3(i),

$$
\begin{aligned}
& s\left(\frac{O_{\varphi+\varphi^{\prime}+\log \delta}(\alpha, \gamma)+\varphi+\varphi^{\prime}+\log 2}{\varphi+\varphi^{\prime}+\log 6}\right) \\
& \quad \geqslant s\left(\min \left\{\frac{O_{\varphi}(\alpha, \beta)+\varphi}{\varphi+\varphi^{\prime}} ; \frac{O_{\varphi^{\prime}}(\beta, \gamma)+\varphi^{\prime}}{\varphi+\varphi^{\prime}}\right\}\right) .
\end{aligned}
$$


Soient $j \in \mathbb{N}, a, a^{\prime} \in 2^{j} .(1+\mathcal{M})$, appliquant le résultat précédent avec $\varphi$ remplacé par $a . \varphi$ et $\varphi^{\prime}$ par $a^{\prime} . \varphi$ on a $s\left(\varphi /\left(\varphi+\varphi^{\prime}\right)\right)=s\left(\varphi^{\prime} /\left(\varphi+\varphi^{\prime}\right)\right)=1 / 2$ et on montre,

$$
\rho_{2 \varphi}(\alpha, \gamma) \geqslant \frac{1}{2} \cdot \min \left\{\rho_{\varphi}(\alpha, \beta) ; \rho_{\varphi}(\beta, \gamma)\right\}
$$

Mais s'il existe $y \in \mathbb{R}, y \geqslant 1$ tel que $O_{y \varphi}(\alpha, \beta) / \varphi$ et $O_{y \varphi}(\beta, \gamma) \notin \mathcal{L}$, on a donc $O_{2 y \varphi+\log \sigma}(\alpha, \gamma) / \varphi \notin \mathcal{L}$, la Proposition 2(i) entraîne $\rho_{2 \varphi}(\alpha, \gamma)-1 \leqslant 1 / 2 .\left(\rho_{\varphi}(\alpha, \gamma)-1\right)$ et on a $\rho_{\varphi}(\alpha, \gamma) \notin s(\mathcal{L})$, d'où $\rho_{\varphi}(\alpha, \gamma)-1=\rho_{\varphi}(\alpha, \gamma)-2$ et $\rho_{2 \varphi}(\alpha, \gamma) \leqslant 1 / 2 . \rho_{\varphi}(\alpha, \gamma)$.

DÉmonstration DU ThÉorème: Il découle immédiatement de la Proposition 2(v) et (iv) que la fonction dam est bien définie, indépendante du choix de $t$ dans $\mathcal{T}$, symétrique et qu'on a $\operatorname{dam}(\tilde{\alpha}, \tilde{\beta})=0$ si et seulement si $\tilde{\alpha}=\tilde{\beta}$.

Posons $u=\tilde{\alpha}, v=\widetilde{\beta}$ et $w=\tilde{\gamma}$, $\operatorname{lorsque} \operatorname{dam}(u, v)$ et $\operatorname{dam}(v, w)$ sont infiniment petits dans $\Gamma_{1}$ il existe $y \in \mathbb{R}, y \geqslant 1$ tel que $O_{y \varphi}(\alpha, \beta) / \varphi$ et $O_{y \varphi}(\beta, \gamma) / \varphi \notin \mathcal{L}$. Le Lemme 4 entraine alors

$$
\operatorname{dam}(u, w) \leqslant \max \{\operatorname{dam}(u, v) ; \operatorname{dam}(v, w)\}
$$

On a, d'après la Proposition 2(o), $\rho(u, v) \geqslant 2$, d'où dam $(u, v) \leqslant 1$.

La fonction dam ainsi introduite permet de définir de façon univoque une topologie séparée sur $\mathbb{C} / \sim$. Soient $u \in \mathbb{C} / \sim$ et $\epsilon \in \Gamma_{1}^{*}$, posons $\mathcal{B}(u, \epsilon)=\{v \in \mathbb{C} / \sim ; \operatorname{dam}(u, v)<$ $\epsilon\}$ et $\overline{\mathcal{B}}(u, \epsilon)=:\{v \in \mathbb{C} / \sim ; \operatorname{dam}(u, v) \leqslant \epsilon\}$.

$D^{\prime}$ après le théorème on a $\mathbb{C} / \sim=\overline{\mathcal{B}}(0,1)$. Il existe une unique topologie sur $\mathbb{C} / \sim$ telle que les ensembles $\mathcal{B}(u, \epsilon)\left(\epsilon \in \Gamma_{1}^{*}\right)$ forment un système fondamental de voisinages de $u \in \mathbb{C} / \sim$. Les ouverts non vides de cette topologie sont les parties $U$ de $\mathbb{C} / \sim$ telles que pour tout $u \in U$ il existe au moins un $\epsilon \in \Gamma_{1}^{*}$ satisfaisant $\mathcal{B}(u, \epsilon) \subset U$. En particulier, d'après le théorème, les parties $\mathcal{B}(u, \epsilon)$, où $\epsilon$ est infiniment petit dans $\Gamma_{1}$, sont à la fois ouvertes et fermées, de même pour les parties $\overline{\mathcal{B}}(u, \epsilon)$. Il en résulte que l'espace $\mathbb{C} / \sim$ muni de cette topologie est totalement discontinu. D'un autre côté, l'espace topologique $\mathbb{C} / \sim$ est séparé car si $u \neq v$ on a dam $(u, v)>0$, il existe $\epsilon \in \Gamma_{1}^{*}$, infiniment petit, tel que $\epsilon<\operatorname{dam}(u, v)$, et on vérifie alors $\mathcal{B}(u, \epsilon) \cap \mathcal{B}(v, \epsilon)=\emptyset$. Ainsi $\mathbb{C} / \sim$ est séparé et tous ses points possèdent un système fondamental de voisinages à la fois ouverts et fermés, autrement-dit $\mathbb{C} / \sim$ est un espace éparpillé (au sens de [3]).

La distance locale détermine une structure uniforme sur $\mathbb{C} / \sim$ (voir $[3$, Section 3$]$ ) dont un système fondamental d'entourage est donné par les parties de $(\mathbb{C} / \sim) \times(\mathbb{C} / \sim)$ de la forme $\{(u, v) ; \operatorname{dam}(u, v)<\epsilon\}\left(\epsilon \in \Gamma_{1}^{*}\right)$. Muni de la topologie et de la structure uniforme ci-dessus, $\mathbb{C} / \sim$ est un espace uniforme, séparé donc complètement régulier (voir [3, Section 4.4]). En fait, l'inégalité ultramétrique locale permet de montrer que tout recouvrement ouvert de $\mathbb{C} / \sim$ admet un recouvrement plus fin par des ensembles 
disjoints de la forme $\mathcal{B}(u, \epsilon)$. On en déduit que $\mathbb{C} / \sim$ est un espace paracompact (voir $[3$, Section 8.20$])$, de dimension de recouvrement nulle.

FAITS.

(i) On a $\operatorname{dam}(\tilde{\alpha}, \tilde{\beta}) \geqslant 1 / 2$ pour tout $(\alpha, \beta) \in \mathbb{C}^{2}$ hors d'un sous-ensemble de $\mathbb{C}^{2}$ Lebesgue-négligeable, de dimension de Hausdorff 2.

(ii) L'espace topologique $\mathbb{C} / \sim$ n'est pas compact.

Démonstration: (i) On suit les démonstration de [10, Théorème 23] ou [4, Théorème 4]. On sait, d'après le théorème (iii), que $\operatorname{dam}(\tilde{\alpha}, \tilde{\beta}) \leqslant 1$, si on a $\operatorname{dam}(\tilde{\alpha}, \tilde{\beta})<1 / 2$ alors $\rho(\alpha, \beta)>4$ et il existe une suite infinie de polynômes $P_{j} \in \mathbb{Z}[X, Y](j \in \mathbb{N})$, sans facteur dans $\mathbb{Z}[X] \cup \mathbb{Z}[Y]$ telle que pour tout $j \in \mathbb{N}$ on ait

$$
\log \left\|P_{j}(\alpha, \beta)\right\| \leqslant-j . t\left(P_{j}\right)^{4} .
$$

On déduit alors du Lemme 2.7 de [9], qu'il existe $\left(\alpha^{\prime}, \beta^{\prime}\right) \in \mathbb{C}^{2}$ tel que $P_{j}\left(\alpha^{\prime}, \beta^{\prime}\right)=0$ et $\log \left\|\left(\alpha-\alpha^{\prime}, \beta-\beta^{\prime}\right)\right\| \leqslant-c_{1} . j . t\left(P_{j}\right)^{3}$ où $\|$.$\| désigne la norme hermitienne habituelle$ sur $\mathbb{C}^{2}\left(\left\|\left(\alpha^{\prime}, \beta^{\prime}\right)\right\|^{2}=\left|\alpha^{\prime}\right|^{2}+\left|\beta^{\prime}\right|^{2}\right)$. On peut aussi supposer, sans perte de généralité, $\|(\alpha, \beta)\| \leqslant 1$.

Si $P \in \mathbb{Z}[X, Y]$ et $x \in \mathbb{R}$, notons $B(\mathcal{Z}(P), x)$ l'ensemble

$$
\begin{array}{r}
\left\{\left(\alpha^{\prime}, \beta^{\prime}\right) \in \mathbb{C}^{2} ; \exists\left(\alpha^{\prime \prime}, \beta^{\prime \prime}\right) \in \mathbb{C}^{2}, P\left(\alpha^{\prime \prime}, \beta^{\prime \prime}\right)=0,\right. \\
\left.\left\|\left(\alpha^{\prime \prime}, \beta^{\prime \prime}\right)\right\| \leqslant 2,\left\|\left(\alpha^{\prime}-\alpha^{\prime \prime}, \beta^{\prime}-\beta^{\prime \prime}\right)\right\|<x\right\}
\end{array}
$$

et posons encore, pour $j, k \in \mathbb{N}^{*}$,

$$
E_{j, k}=\bigcup B\left(\mathcal{Z}(P), \exp \left(-c_{1} \cdot j . t(P)^{3}\right)\right), E_{j}=\bigcup_{k \geqslant 1} E_{j, k} \quad \text { et } \quad E=\bigcap_{j \geqslant 1} E_{j}
$$

où la première réunion porte sur tous les polynômes $P \in \mathbb{Z}[X, Y]$, sans facteur dans $\mathbb{Z}[X] \cup \mathbb{Z}[Y]$ et satisfaisant $k \leqslant t(P)<k+1$. On a $E_{1} \supset E_{2} \supset \ldots$ et $(\alpha, \beta) \in E$ dès que $\|(\alpha, \beta)\| \leqslant 1$ et $\operatorname{dam}(\tilde{\alpha}, \widetilde{\beta})<1 / 2$, vérifions que $E$ est de mesure de Lebesgue $\mu(E)$ nulle. Le nombre de termes dans la réunion définissant $E_{j, k}$ est majoré par $\exp \left(c_{2} k^{\mathbf{s}}\right)$, tandis que la mesure de Lebesgue de chacun des ensembles est majorée par $c_{3} \cdot d^{0} P \cdot \exp \left(-c_{1} \cdot j \cdot t(P)^{3}\right) \cdot$ Ainsi

et on en déduit

$$
\mu\left(E_{j, k}\right) \leqslant c_{3} \cdot(k+1) \cdot \exp \left(-\left(c_{1} j-c_{2}\right) \cdot k^{3}\right),
$$

$$
\mu\left(E_{j}\right) \leqslant \sum_{k \geqslant 1} \mu\left(E_{j, k}\right) \leqslant c_{3} \cdot \sum_{k \geqslant 1}(k+1) \cdot \exp \left(-\left(c_{1} j-c_{2}\right) \cdot k^{3}\right) \leqslant c_{4} \cdot \exp \left(-c_{1} \cdot j\right),
$$


d'où $\mu(E)=0$, car $\mu(E) \leqslant \mu\left(E_{j}\right)$ pour tout $j \in \mathbb{N}^{*}$. On montre de même que la $s$-mesure de Hausdorff de $E$ est nulle pour $s>2$ (voir [1, Section 4]). Mais, d'un autre côté, $E$ contenant la diagonale de $\mathbb{C}^{2}$ est de dimension de Hausdorff $\geqslant 2$.

(ii) Comme, d'après (i), $\operatorname{dam}(\tilde{\alpha}, \tilde{\beta}) \geqslant 1 / 2$ pour $(\alpha, \beta)$ hors d'un ensemble Lebesgue-négligeable de $\mathbb{C}^{2}$, on construit une suite $u_{0}=0, u_{1}, \ldots$ d'éléments de $\mathbb{C} / \sim$ telle que $u_{n} \in \bigcap_{i=0}^{n-1}\left\{v ; \operatorname{dam}\left(u_{i}, v\right) \geqslant 1 / 2\right\}$. En effet, cette dernière intersection contient les classes de presque tous les nombres complexes. On a donc $\operatorname{dam}\left(u_{i}, u_{j}\right)=0$ si $i=j$ et $\geqslant 1 / 2$ si $i \neq j$, et comme dans un espace compact le filtre associé à toute suite doit admettre au moins un point adhérent on en déduit que $\mathbb{C} / \sim$ n'est pas compact.

EXEMPLE: L'ultrafiltre $\mathcal{U}$ étant fixé on suppose $\varphi=i d$ et on considère $\gamma \in \mathcal{C}$, $\gamma \geqslant 2$. Posons $a_{0}=1, a_{k}=\prod_{i=1}^{k-1}\left(2 a_{i} \gamma\left(2 a_{i}\right)\right)$ pour $k \geqslant 1$ et

$$
b_{n}=\sum_{k=1}^{2 n} a_{k}, \quad b_{n}^{\prime}=\sum_{k=1}^{2 n+1} a_{k},
$$

on a $b_{n} \leqslant 2 . a_{2 n}$ et $b_{n}^{\prime} \leqslant 2 . a_{2 n+1}$. On introduit les nombres réels suivants associés aux suites $\left(b_{n}\right)$ et $\left(b_{n}^{\prime}\right)$ :

$$
\theta=\sum_{n \geqslant 1} 2^{-\left[e^{b_{n}}\right]} \text { et } \theta^{\prime}=\sum_{n \geqslant 1} 2^{-\left[e^{b_{n}^{\prime}}\right]}
$$

On vérifie, pour $n$ assez grand, les propriétés suivantes

$$
\begin{aligned}
& O(j \mid \theta) / j \geqslant \frac{b_{n+1}-b_{n}-c}{b_{n}^{\prime}} \geqslant \frac{a_{2 n+2}+a_{2 n+1}-c}{2 . a_{2 n+1}} \geqslant \gamma(i)-c \text { pour } j \in\left[b_{n}, b_{n}^{\prime}\right] \\
& O\left(j \mid \theta^{\prime}\right) / j \geqslant \frac{b_{n+1}^{\prime}-b_{n}^{\prime}-c}{b_{n+1}} \geqslant \frac{a_{2 n+3}+a_{2 n+2}-c}{2 . a_{2 n+2}} \geqslant \gamma(j)-c \text { pour } j \in\left[b_{n}^{\prime}, b_{n+1}[\right.
\end{aligned}
$$

où $c>0$ est un réel indépendant de $n$ et $j$. Mais, pour tout $m \in \mathbb{N}$, un des ensembles $\bigcup_{n \geqslant 1}\left[2^{-m} \cdot b_{n}, 2^{-m} \cdot b_{n}^{\prime},\left[\cap \mathbb{N}\right.\right.$ ou $\bigcup_{n \geqslant 1}\left[2^{-m} \cdot b_{n}, 2^{-m} \cdot b_{n+1}[\cap \mathbb{N}\right.$ appartient à $\mathcal{U}$ et donc, avec $\varphi_{m}=2^{m} . i d$, on a $s\left(O_{\varphi_{m}}(\theta) / \varphi_{m}\right) \geqslant s(\gamma)$ ou $s\left(O_{\varphi_{m}}\left(\theta^{\prime}\right) / \varphi_{m}\right) \geqslant s(\gamma)$. De même, l'ensemble des $m$ tels que la première éventualité se présente appartient à $\mathcal{U}$ ou c'est l'ensemble des $m$ tels que la seconde éventualité se présente qui appartient à $\mathcal{U}$. On en déduit $\rho_{\varphi}(\theta)$ ou $\rho_{\varphi}\left(\theta^{\prime}\right) \geqslant s(\gamma)$ dans $\Gamma$ avec $\varphi=\varphi_{0}=i d$. Enfin, on vérifie (voir [4], par exemple) que ni $\theta$, ni $\theta^{\prime}$ n'est algébrique. Ainsi, pour tout élément $\gamma$ de $\Gamma_{1}$ il existe une classe non nulle de $\mathbb{C} / \sim$ d'ordre de transcendance (relatif à $\mathcal{U}$ ) $\geqslant \boldsymbol{\gamma}$.

REMARQUe 10. Lorsque $\alpha \in \mathbb{C}$ et $\varphi$ est de la forme $\varphi(j)=f . e^{j} ; j \in \mathbb{N}, e, f \in \mathbb{R}_{+}^{*}, e>$ 1 , le diagramme de la fonction $O_{\varphi}(j \mid \alpha) / \varphi(j)$ contre $j$ est un diagramme en dents de scie exponentielles. On a un ensemble de sauts

$$
E_{\text {saut }}=\left\{j \in \mathbb{N} ; O_{\varphi}(j-1 \mid \alpha)<O_{\varphi}(j \mid \alpha)\right\},
$$


en entre chaque saut la fonction $O_{\varphi}(j \mid \alpha) / \varphi(j)$ doit nécessairement retomber selon une loi exponentielle décroissante à une valeur $\leqslant 1+\epsilon(j)$ (où $\epsilon \in \mathbb{R}^{\mathbf{N}}$ définit un élément de $\mathcal{M}$ ). L'ordre de transcendance habituellement défini dans la littérature est la limite supérieure de ces dents de scie alors que nous avons opté pour une limite selon un ultrafiltre. En particulier, on notera qu'il ne suffit pas que la limite supérieure soit $+\infty$ pour que l'ordre de transcendance que nous avons défini soit infiniment grand, il faut encore que les sauts correspondants aux très bonnes approximations soient assez "fréquents" selon l'ultrafiltre choisi.

L'exemple précédent la remarque 10 montre que la distance dam que nous avons définie et la topologie déduite ne sont pas discrètes.

Question 1. Pour $u \in \mathbb{C} / \sim$ fixé que peut-on dire de l'ensemble des $\gamma \in \Gamma^{*}$ (i.e. $\gamma \in \Gamma, \gamma \neq 0)$ tels que $\gamma=\operatorname{dam}(u, v)$ pour au moins un $v$ dans $\mathbb{C} / \sim$ ?

QUESTION 2. L'espace uniforme $\mathbb{C} / \sim$ est-il complet?

\section{RÉFÉRENCES}

[1] F. Amoroso, 'On the distribution of complex numbers according to their transcendance types', Ann. Mat. Pura Appl. (4) 151 (1988), 359-368.

[2] A. Baker, Transcendental number theory (Cambridge Univ. Press, 1979).

[3] N. Bourbaki, Topologie générale, fasc. de résultats (Hermann, 1964).

[4] A. Durand, 'Quatre problèmes de Mahler sur la fonction ordre d'un nombre transcendant', Bull. Soc. Math. France 102 (1974), 365-377.

[5] A. Durand, 'Fonction $\theta$-ordre et classification de $\mathbb{C}^{p}$, C.R. Acad. Sci. Paris Sér. A Math. 280 (1975), 1085-1088.

[6] Y. Hellegouarch, 'Introduction à la théorie des nombres non-standard', in Séminaire de Théorie des Nombres, 1991 (Publ. Univ. Caen, 1991), pp. 10.

[7] T. Lindstrom, 'An invitation to non-standard analysis', in Nonstandard analysis and its applications, (N. Cutland, Editor), London Math. Soc. Stud. Texts 10 (Cambridge Univ. Press, 1988), pp. 1-105.

[8] K. Mahler, 'On the order function of a transcendental number', Acta Arith. 18 (1971), 63-76.

[9] P. Philippon, 'Critères pour l'indépendance algébrique', Inst. Hautes Etudes Sci. Publ. Math. 64 (1986), 5-52.

[10] T. Schneider, Einführung in die Transzendenten Zahlen, Grundlehren Math. Wiss. 81 (Springer, Berlin, Heidelberg, New York, 1957). Introduction aux nombres transcendants (Gauthier-Villars, Paris, 1959).

[11] V.G. Sprindzhuk, 'Achievements and problems in diophantine approximation theory', Uspekhi Mat. Nauk 35 (1980), 3-68. Russian Math. Surveys 35(4) (1980), 1-80.

[12] M Waldschmidt, Nombres transcendants, Lecture Notes in Math. 402 (Springer, Berlin, Heidelberg, New York, 1974). 
[13] Yu KunRui, 'A generalization of Mahler's classification to several variables', J. Reine Angew. Math. 377 (1987), 113-126.

Probèmes Diophantiens

Université $P$. et $M$. Curie

T.45-46, 5 ème ét.

75252 Paris, Cedex 05

France France 